\title{
Efeitos de um programa de reabilitação fisioterapêutica após videoartroscopia de impacto femoroacetabular
}

\author{
Effects of a physiotherapy rehabilitation \\ program after arthroscopy for \\ femoroacetabular impact
}

FisiSenectus. Unochapecó Ano 4, n. 1 - Jan/Jun. 2016 p. $42-50$

Janesca Mansur Guedes. janescaguedes@yahoo.com.br

Doutora em Ciências da Saúde. Universidade Regional Integrada do Alto Uruguai e das Missões (URI) - Campus Erechim/RS.

Alexandre Marek. alexandremarek@yahoo.com.br

Especialista em Fisioterapia em Ortopedia e Traumatologia. Universidade de Passo Fundo-RS.

Deyse Mara Piva. deysepiva@hotmail.com

Especialista em Fisioterapia em Ortopedia e Traumatologia.

\section{Resumo}

Introdução: 0 impacto femoroacetabular (IFA) é considerado uma condição de dor no quadril jovem acompanhado de perda de amplitude de movimento (ADM) e alterações degenerativas. A técnica artroscópica do IFA visa modificar anatomicamente o fêmur proximal e o acetábulo, assim como restaurar lesões condrais e labrais, com o intuito de diminuir o contato do fêmur contra o rebordo acetabular, e, dessa forma, melhorar a mobilidade do quadril e prevenir a osteoartrose precoce. 0 tratamento fisioterapêutico após a intervenção cirúrgica visa à boa recuperação funcional do paciente para o retorno ao seu nível de atividade. Objetivo: avaliar os efeitos de um programa de reabilitação fisioterapêutica na ADM, força, percepção da dor e funcionalidade, dos pacientes submetidos à videoartroscopia de quadril para tratamento de IFA. Materiais e métodos: Participaram do estudo seis pacientes, aos quais foi aplicado o programa de reabilitação após a videoatrtroscopia de quadril por IFA do Instituto de Ortopedia e Traumatologia (IOT) - Hospital do Trauma de Passo Fundo/RS. Foram analisadas as variáveis ADM, força muscular, Escala Visual Analógica (EVA) e a funcionalidade através do questionário Harris Hip Score modificado na $1^{\mathrm{a}}, 4^{\mathrm{a}}$ e $8^{\mathrm{a}}$ semana de pós-operatório. Conclusão: Ao final do estudo chegou-se à conclusão que houve melhora progressiva nas avaliações em todas as variáveis analisadas.

\section{Palavras-chave}

Lesões do quadril; Impacto femoroacetabular; Reabilitação. 


\begin{abstract}
Introduction: Femoroacetabular impingement (IFA) is considered a pain condition in young hip accompanied by loss of range of motion (ROM) and degenerative changes. The IFA arthroscopic technique seeks to change anatomically proximal femur and acetabulum, as well as restore chondral and labral lesions, in order to decrease the contact of the femur against the acetabular rim, and thus improve hip mobility. The physical therapy after surgery aims to functional recovery of the patient to return to previous activity level. Objective: This study aimed to evaluate the effect of a physical therapy rehabilitation program in patients undergoing hip videoarthroscopy to IFA treatment. Materials and methods: Six patients where evaluated and the hip videoarthroscopy rehabilitation program for IFA Institute of Orthopedics and Traumatology (IOT) - Trauma Hospital in Passo Fundo R / S was apllied. The follow variables were analyzed: ROM, muscle strength, Visual Analogue Scale (VAS) and function through the questionnaire Harris Hip Score modified in the 1st, 4th and 8th week after surgery. Conclusions: At the end of the study reached the conclusion that there was progressive improvement in assessments on all variables.
\end{abstract}

\title{
Keywords
}

Hip Injuries; Femoroacetabular impingment; Rehabilitation.

\section{Introdução}

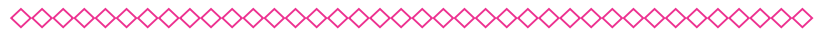

O quadril consiste na articulação entre o acetábulo hemisférico e a grande cabeça do fêmur com formato esferoidal, denominada de articulação coxofemoral. Essa articulação tem características anatômicas importantes para que ocorra a estabilidade durante a ortostase, a marcha e a corrida ${ }^{1}$. 0 impacto femoroacetabular (IFA) é causado por conflitos entre a junção femoral cabeça/colo, o acetábulo periférico e a borda acetabular. Dois tipos de IFA foram identificados: pincer e came. 0 tipo pincer ocorre pelo impacto repetitivo entre 0 lábio acetabular e a transição cabeça/colo femoral nos casos de excesso de cobertura acetabular. No tipo came ocorre a perda da concavidade da transição cabeça/colo femoral, que determina impacto na porção anterosuperior da cartilagem acetabular. Muitas vezes, a intervenção cirúrgica torna-se a única opção para o alívio da dor ${ }^{2,3}$.

Um dos tratamentos propostos para o tratamento do IFA é a artroscopia, por apresentar tempo de reabilitação reduzido e facilitar o acesso à articulação do quadril ${ }^{4}$. A reabilitação imediata após a cirurgia do IFA visa o controle da dor e controle da inflamação pós-operatória. 0 tratamento da fisioterapia deve completar a boa recuperação funcional dos pacientes, buscando normalizar amplitude de movimento, força e resistência, sem agravar os sintomas do paciente, e o retorno à prática esportiva, ${ }^{5,6}$.

O objetivo deste estudo foi avaliar o efeito de um programa de reabilitação fisioterapêutica na amplitude de movimento, força muscular, percepção da dor e funcionalidade dos pacientes submetidos à videoartroscopia de quadril para o tratamento do impacto femoroacetabular.

\section{Materiais e métodos}

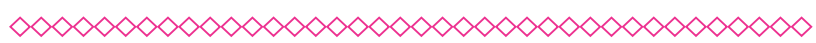

Este estudo possui um caráter intervencionista, com corte longitudinal e quantitativo. Fizeram parte da pesquisa seis pacientes que foram submetidos à videoartroscopia de IFA. As avaliações foram realizadas no Serviço de Fisioterapia do IOT - Hospital do Trauma de Passo Fundo/RS no ano de 2015. Por critério de inclusão foram selecionados os pacientes que realizaram o procedimento cirúrgico no IOT e que iniciaram a reabilitação até a terceira semana do pós-operatório e ainda, que realizaram todas as fases da reabilitação no Serviço de Fisioterapia do IOT e concordaram em assinar o termo de consentimento livre e esclarecido (TCLE). Foram excluídos os pacientes que faltaram mais de duas sessões de fisioterapia durante o período da reabilitação e aqueles que expressaram o desejo em não continuar fazendo parte do processo de pesquisa 
ou, ainda, os que realizaram alguma fase da reabilitação em algum outro Serviço de fisioterapia.

Os atendimentos foram realizados por diferentes fisioterapeutas e tiveram duração de cerca de uma hora, com frequência de três vezes por semana durante dois meses, totalizando 24 sessões. Os pacientes foram submetidos a três avaliações no decorrer de sua reabilitação. A primeira avaliação foi realizada no primeiro dia da reabilitação, a segunda na $4^{\mathrm{a}}$ semana, e a terceira e última na $8^{\mathrm{a}}$ semana de reabilitação. Foram avaliadas a ADM, força muscular, intensidade da dor e a funcionalidade do quadril operado. Todas as avaliações foram realizadas pelo pesquisador responsável. O protocolo de tratamento está apresentado na Figura 1.

A ADM foi mensurada através da goniometria ativa do quadril. Foram avaliados os movimentos de flexão, extensão, abdução, adução, rotação externa e rotação interna ${ }^{7}$.

A avaliação da percepção dor foi realizada através da Escala Visual Analógica (EVA), através de uma linha com as numerações de (0) a (10). Em uma extremidade da linha foi marcado o número zero (0), que equivale a 'nenhuma dor' e na outra extremidade o número dez (10) que é considerado 'dor excessiva'. Foi solicitado que o paciente marcasse na linha a percepção da dor que estava sentindo naquele momento.

A força muscular foi avaliada através dos testes manuais descritos por Kendall ${ }^{8}$, que descreve ao realizar as provas de força muscular um escore de zero (0) a cinco (5), onde (5) representa que o paciente apresenta força normal, (4) apresenta movimento ativo contra a gravidade e resistência, (3) apresenta movimento ativo contra a gravidade, (2) movimento ativo com eliminação da gravidade, (1) contração visível sem movimento do segmento e (0) nenhuma contração muscular visível. Os grupos musculares avaliados foram os flexores de quadril, extensores, abdutores, adutores, rotadores externos e rotadores internos.

Para avaliação da funcionalidade foi aplicada a versão brasileira do questionário Harris Hip Score, o qual é um instrumento padronizado e adaptado à cultura brasileira para avaliação da qualidade de vida de pacientes com afecção do quadril. Pela necessidade de avaliar os resultados artroscópicos, avalia-se a dor que corresponde a 44 pontos e função correspondente a 47 pontos. A multiplicação por constante " 1,1 " resulta no escore total possível de 100 pontos $^{9,10}$.

Os dados obtidos através das avaliações foram armazenados e tabulados no Software Microsoft ${ }^{\circledR}$ Excel, e as variáveis foram apresentadas em média e desvio padrão. Foi utilizado ANOVA one-way, seguida pelo teste post-hoc de Tukey para comparar as três avaliações, com auxílio do software GraphPad Prism 6, e, considerado significativo um $p<0,05$.

Este estudo, em observância às diretrizes da Resolução CNS 466/12 do Conselho Nacional da Saúde do Ministério da Saúde, foi aprovado pelo Comitê de Ética em Pesquisa da URI - Campus de Erechim e registrado sob número do CAAE 47788115.9.0000.5351.

\section{Resultados e discussão}

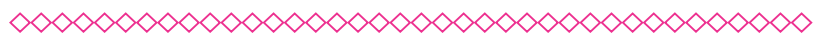

Foram incluídos no presente estudo seis pacientes, três em pós-operatório de videoartroscopia por IFA no quadril direito e três em pós-operatório de videoartroscopia por IFA no quadril esquerdo.

Este estudo foi composto por cinco pacientes do sexo masculino, com idade entre 28 e 42 anos, sendo a média de 35,4 $\pm 5,4$ anos e um paciente do sexo feminino com 47 anos; $50 \%$ dos pacientes apresentaram lesão no quadril direito e $50 \%$ com lesão no quadril esquerdo.

A prevalência da lesão no sexo masculino em comparação ao sexo feminino na amostra desta pesquisa condiz com a literatura, onde afirma que esta condição é encontrada mais comumente em pacientes jovens do sexo masculino ${ }^{11}$. Também, a faixa etária dos pacientes com IFA presente neste estudo corresponde à literatura estudada ${ }^{3,5,11,13}$, onde relata-se que este tipo lesão acomete principalmente pacientes entre as metades da segunda e quarta décadas de vida, assim como os indivíduos submetidos à artroscopia para tratar o IFA são suscetíveis por se originar de uma população mais ativa fisicamente.

A Figura 2 apresenta os dados referentes à avaliação da ADM do quadril antes de começar o 
tratamento fisioterapêutico (Avaliação 1), na quarta semana de reabilitação (Avaliação 2) e no final do tratamento (Avaliação 3).

Os resultados apresentados na Figura 5.2 demonstram um aumento significativo da ADM de flexão $(p=0,0007)$, extensão $(p=0,02)$, abdução $(p=0,0006)$, adução $(p=0,0005)$, rotação externa $(p=0,0025)$ e rotação interna $(p=0,0280)$ do quadril na avaliação final quando comparados à avaliação inicial. As ADM's de flexão, abdução e adução apresentaram já na $4^{a}$ semana aumento significativo de ADM quando comparado à avaliação inicial.

A progressão da ADM segue diretrizes relativamente básicas, nas primeiras duas semanas se considera uma fase de proteção, começa com o tolerado pelo paciente, com cautela exercida para evitar excessiva flexão do quadril, rotação interna e abdução, precisa evitar uma força excessiva para a região do colo do fêmur no início do processo de reabilitação, assim como o movimento de rotação interna com o quadril em uma posição de flexão deve ser abordado com cautela ${ }^{5}$. Uma vez que a inflamação diminui, a ADM passiva completa deve ser incentivada, isto geralmente ocorre entre duas a quatro semanas após a cirurgia. 0 alongamento dos tendões pode ser iniciado de duas a três semanas após a cirurgia. Ao final da quarta semana após a cirurgia, o alongamento suave de toda musculatura na região da articulação do quadril deve ser iniciado e progredir como tolerado ${ }^{5}$.

Exercícios de ADM e alongamento são importantes para melhorar as articulações durante o período pós-cirúrgico ou em casos de pacientes que sofrem de patologias crônicas. Eles também são importantes para aumentar a flexibilidade, para evitar a formação de aderência entre os tecidos moles e ossos, fibrose periarticular, para remodelar e melhorar a extensibilidade do músculo e outros tecidos, para ajudar a evitar o agravamento das lesões, sejam elas de articulações, músculos, tendões e ligamentos.

A Figura 3 demonstra os resultados da avaliação da força muscular de flexão, extensão, abdução, adução, rotação externa e rotação interna.

Os resultados apresentados da força muscular (Figura 3) demonstram um aumento significativo da força muscular dos flexores de quadril $(p=0,02)$, extensores $(p=0,007)$, adutores $(p=0,02)$, rotação externa $(p=0,04)$ e rotação interna $(p=0,004)$ na avaliação final, quando comparados à avaliação inicial. Os resultados da flexão, bem como da adução apresentaram já na $4^{a}$ semana aumento estatístico de força muscular quando comparados à avaliação inicial.

Seguindo o protocolo utilizado neste estudo, as atividades de força muscular suaves são iniciadas no dia seguinte à cirurgia. A progressão da atividade de força geralmente é iniciada em duas semanas após a cirurgia com uma atenção especial ao recrutamento do músculo glúteo médio ${ }^{14}$. Os indivíduos submetidos a procedimentos concomitantes para liberar a banda iliotibial e tendão iliopsoas devem atrasar o início de atividades específicas como a elevação da perna estendida para evitar a irritação do tecido de cicatrização ${ }^{5}$, nos pacientes que têm a banda iliotibial liberada, a abdução em decúbito lateral deve ser adiada por cerca de quatro semanas, pois o início dessas atividades muito cedo no processo de reabilitação pode resultar em tendinite no pós-operatório. O levantamento de peso e aumento das atividades são iniciados entre a quarta e a sexta semana após a cirurgia. As atividades gerais de força devem incidir sobre os quadríceps, isquiotibiais e glúteos ${ }^{5}$. A Figura 4 demonstra a percepção da dor dos pacientes através da escala visual analógica (EVA).

Os resultados apresentados na Figura 5.4 demonstram uma redução significativa da percepção da intensidade da dor na quarta semana e oitava semana quando comparados a avaliação inicial $(p=0,003)$. Em relação à intensidade da dor, na avaliação inicial, 66,67 \% dos pacientes relataram sentir 'pouca dor', representada numericamente de (1) a (3) na escala EVA. Descreveram estar com 'razoável ou muita dor' 33,33 \% do total da amostra. Na avaliação realizada na quarta semana houve uma queda importante do relato de dor. Todos os pacientes, totalizando $100 \%$ da amostra classificaram a dor com pontuação de (1) a (3), ou seja, pouca dor, sendo que destes, $50 \%$ identificaram a pontuação mínima da escala (1). Por fim, na terceira e última avaliação, houve uma queda ainda maior em relação a queixas com o desconforto da dor. Da amostra total, 50 \% não relataram a ocorrência de dor, e os demais 50 \% dividiram-se entre a intensidade de (1) a (2), sendo 33,33\% para a 
intensidade mínima (1) e 16,67\% com a pontuação (2) na escala EVA.

A mensuração da dor é extremamente importante no ambiente clínico, pois se torna impossível manipular um problema desta natureza sem ter uma medida sobre a qual basear o tratamento ou a conduta terapêutica. Sem tal medida, torna-se difícil determinar se um tratamento é necessário, se o tratamento prescrito é eficaz, ou mesmo quando deve ser interrompido um dado tratamento. Uma mensuração apropriada da dor torna-se possível determinar se os riscos de um dado tratamento superam os danos causados pelo problema clínico e, também, permite escolher qual é o melhor e o mais seguro entre diferentes tipos de conduta terapêutica. Ao lado disso, pode-se fazer um meIhor acompanhamento e análise dos mecanismos de ação de diferentes drogas farmacológicas. Em outras palavras, é possível aperfeiçoar a escolha ${ }^{15}$.

A Figura 5 demonstra os resultados obtidos na aplicação do questionário Harris Hip Score Modificado.

Os resultados apresentados na Figura 5 demonstram um aumento significativo na funcionalidade na quarta semana e na oitava semana quando comparados a primeira semana $(p=0,0004)$. $\mathrm{Na}$ totalidade dos aspectos avaliados, dor, função, mobilidade e deformidade e Harris total, somente a deformidade manteve-se com a mesma média, mantendo-se em zero.

\section{Considerações finais}

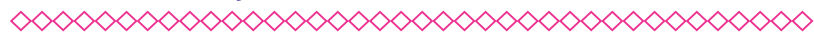

A pesquisa da Literatura através de teorias, conceitos e estudos já realizados por autores especialistas no tema, bem como a pesquisa de campo que proporcionou a aplicação destes conhecimentos na prática permitiram o embasamento necessário para o alcance dos objetivos da pesquisa. Ao término do estudo concluiu-se que o protocolo utilizado para a reabilitação dos pacientes, num período de oito semanas, submetidos à videoartroscopia nas lesões de IFA, foi eficaz para melhorar a amplitude de movimento, a força muscular, a percepção da dor e a funcionalidade do quadril.

\section{Referências}

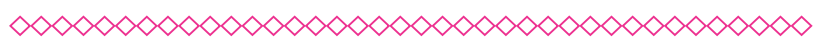

1. Neumann D. Cinesiologia do aparelho músculoesquelético: fundamentos para reabilitação. 2. ed. Rio de Janeiro: Elsevier; 2011.

2. Ganz R, Parvizi J, Beck M, Leunig M, Nötzli H, Siebenrock KA. Femoroacetabular impingement: a cause for osteoarthritis of the hip. Clin Orthop Relat Res. 2003;(417):112-20.

3. Sampson TG. Arthroscopic treatment of femoroacetabular impingement. The American Journal of Orthopedics. 2008;379(12):608-12.

4. Ross BD, Ross MV, Camisa Junior A, Lima MEU, Giboski DP, Martins LS. Abordagem extracapsular para tratamento artroscópico de impacto femoroacetabular: resultados clínicos, radiográficos e complicações. Rev Bras Ortop. 2015;50(4):430-37.

5. Enseki KR, Martin R, Kelly BT Rehabilitation after arthroscopic decompression for femoroacetabular impingement. Clinics in Sports Medicine. 2010;29:247-55.

6. Sa'nchez DCL, Girone's RMC, Santamaría CFJ, Herna'ndez GMC, Alcove A.Fisioterapia tras primera intervencio'n de osteoplastia miniinvasiva en atrapamiento femoroacetabular en un hospital universitario. Fisioterapia. 2010;32(6):291-95.

7. Marques A. Manual de goniometria. 2. ed. Barueri, SP: Manole; 2003.

8. Kendall F, Kendall E. Músculos: provas e funções. 4. ed. São Paulo: Manole; 1995.

9. Harris WH. Etiology of osteoarthritis of the hip. Clin Orthop Relat Res. 1986; 213:20-33.

10. Guimarães RP, Alves RPL, Azuaga TL, Ono NK, Honda E, Polesello GC, Ricioli Junior W, Ueno LU, Carvalho NAA. Tradução e adaptação transcultural do "Harris Hip Score modificado por Byrd". Acta ortopédica brasileira. 2010;18(6):339-42.

11. Parvizi J, Leunig M, Ganz R. Femoroacetabular Impingement. The Journal of the American Academy of Orthopaedic Surgeons. 2007;15(9):561-70. 
12. Tannast M, Siebenrock KA, Anderson SE. Femoroacetabular Impingement: Radiographic Diagnosis-What the Radiologist Should Know. American Journal of Roentgenology. 2007; 188:1540-52.

13. Mauro CS, Voos JE, Kelly BT. Femoroacetabular Impingement Surgical Techniques. Operative Techniques in Orthopaedics. 2010;20:223-30.
14. Frasson VB, Moraes AB, Torresan A, Crestani $M$, Fortes PDG, Telökend MA, et al. Fisioterapia no pós-operatório de correção artroscópica do impacto femoroacetabular. 2015;8(3):156-168.

15. Silva A, Ribeiro Filho NP. A dor como um problema psicofísico. Rev Dor. 2011; 12(2):138-51. 


\section{Anexos}

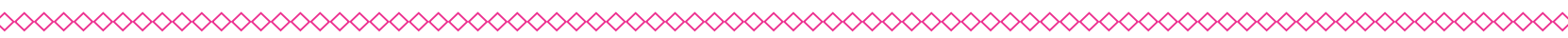

Figura 1 - Protocolo de Reabilitação de Pós-Operatório de Impacto Femoroacetabular (Videoartroscopia) do Serviço de Fisioterapia do IOT Hospital do Trauma, 2016

\section{DESCRIÇÃO DO PROCEDIMENTO:}

\section{Semanas 1 e 2 de PO}

* Alongamentos analíticos dos adutores do quadril, isquiotibiais e quadríceps (mantidos por 30" cada);

* Mobilização articular para decoaptação longitudinal, decoaptação lateral e flexão do quadril;

* Exercícios ativos isotônicos para extensores do quadril, quadríceps e isquiotibiais (3 séries de 10 repetições, com estabilização do CORE);

* Exercícios isométricos para adutores e abdutores do quadril (3 séries de 10 repetições, com estabilização do CORE);

* Fortalecimento para musculatura abdominal (3 séries de 10 repetições);

* Treino de marcha com descarga parcial de peso;

* Eletroterapia (analgesia) se necessário.

\section{Semanas 3 e 4 de PO}

* Alongamentos analíticos dos adutores do quadril, isquiotibiais e quadríceps (mantidos por 30" cada);

* Mobilização articular para decoaptação longitudinal, decoaptação lateral e flexão do quadril;

* Exercícios ativos isotônicos para extensores do quadril, quadríceps e isquiotibiais (3 séries de 10 repetições, com estabilização do (ORE);

* Exercícios isométricos para adutores e abdutores do quadril (3 séries de 10 repetições, com estabilização do CORE);

* Fortalecimento para musculatura abdominal (3 séries de 10 repetições);

* Treino de marcha com descarga parcial de peso;

* Descarga de peso (3 séries de 10 repetições);

* Bicicleta estacionária vertical (durante 10 minutos);

* Eletroterapia (analgesia) se necessário.

\section{Semanas 5 a 8 de PO}

* Alongamentos analíticos dos adutores e abdutores do quadril, isquiotibiais e quadríceps (mantidos por 30" cada);

* Mobilização articular para decoaptação longitudinal, decoaptação lateral, rotação interna, totação externa e flexão do quadril;

* Exercícios ativos isotônicos contra resistidos para extensores do quadril, quadríceps e isquiotibiais ( 3 séries de 10 repetições, com estabilização do CORE);

* Exercícios isométricos para adutores e abdutores do quadril (3 séries de 10 repetições, com estabilização do CORE);

* Fortalecimento para musculatura abdominal (3 séries de 10 repetições);

* Treino de marcha;

* Descarga de peso (3 séries de 10 repetições);

* Agachamento bipodal (3 séries de 10 repetições);

* Fortalecimento do tríceps sural (3 séries de 10 repetições);

* Bicicleta estacionária vertical (durante 10 minutos);

* Eletroterapia (analgesia) se necessário.

Fonte: Elaborado pelo fisioterapeuta Alexandre Marek, revisado pelo Serviço de Cirurgia do Quadril IOT 
Figura 2 - Avaliação da amplitude de movimento do quadril, $2016^{1}$

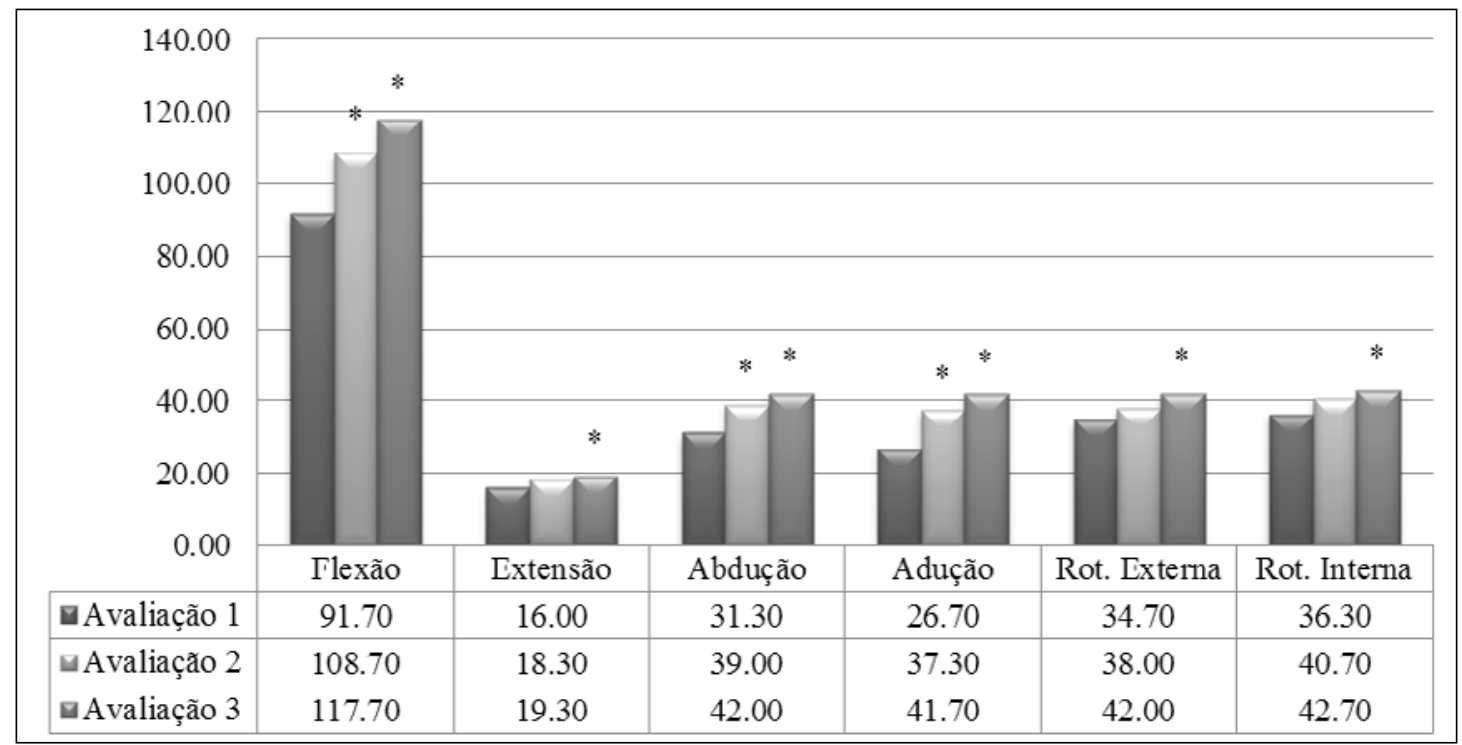

Fonte: Elaborado pelos autores

(clique para voltar ao texto)

Figura 3 - Avaliação da força muscular de Flexão, Extensão,

Abdução, Adução, Rot. Externa e Rot. Interna, 2016²

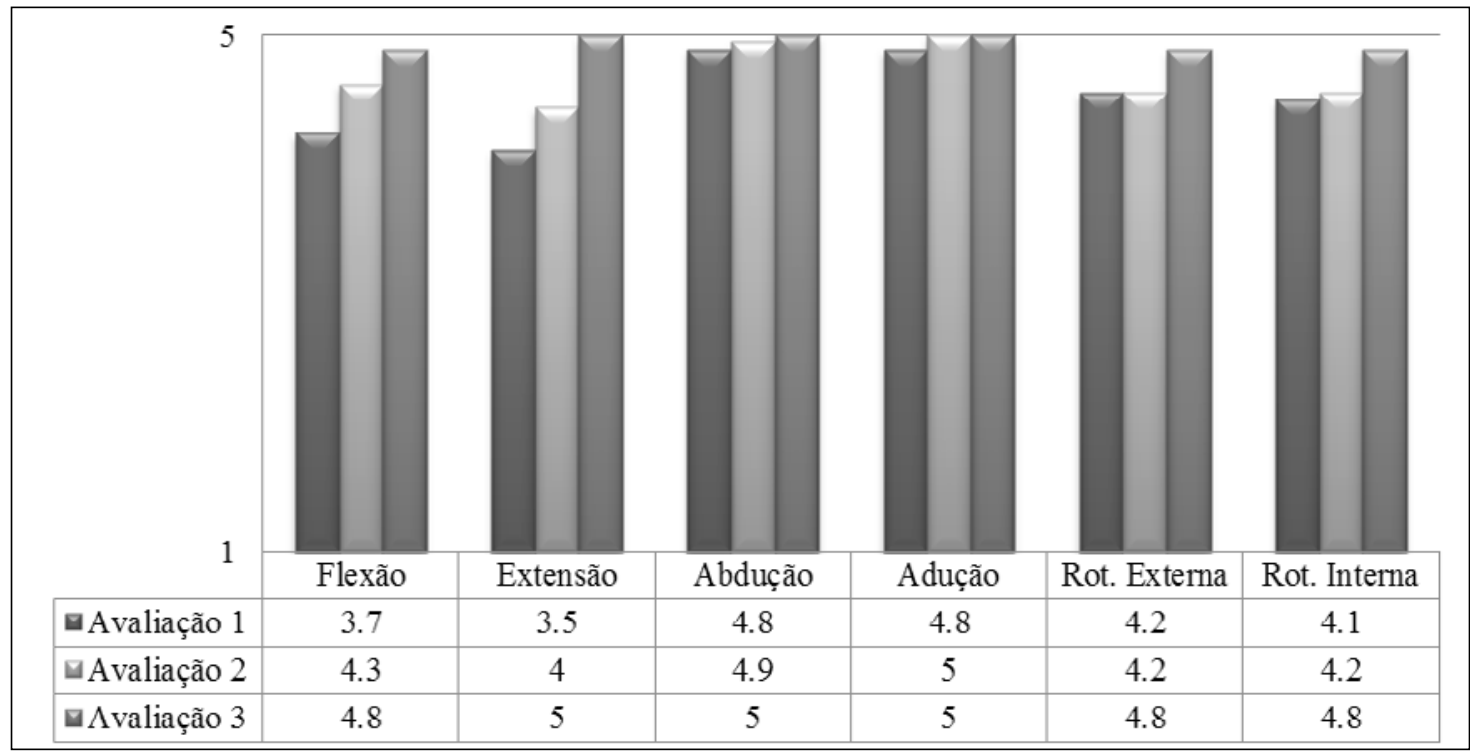

Fonte: Elaborado pelos autores

(clique para voltar ao texto)

\footnotetext{
${ }^{1}$ Valores apresentados com a média da ADM de flexão, extensão, abdução, adução, rot. externa e rot. interna na $1^{a}$ semana (avaliação 1),4a semana (avaliação 2) e na $8^{a}$ semana de pós-operatório (avaliação 3). *representa diferença estatística em relação à primeira avaliação para o mesmo movimento.

${ }^{2}$ Valores apresentados com a média da força muscular de flexão, extensão, abdução, adução, rot. externa e rot. interna na $1^{\text {a }}$ semana (avaliação 1 ), $4^{\text {a }}$ semana (avaliação 2) e na $8^{a}$ semana de pós-operatório (avaliação 3). *representa diferença estatística em relação à primeira avaliação para o mesmo movimento.
} 
Figura 4 - Percepção da intensidade da dor através da escala visual analógica (EVA), $2016^{3}$

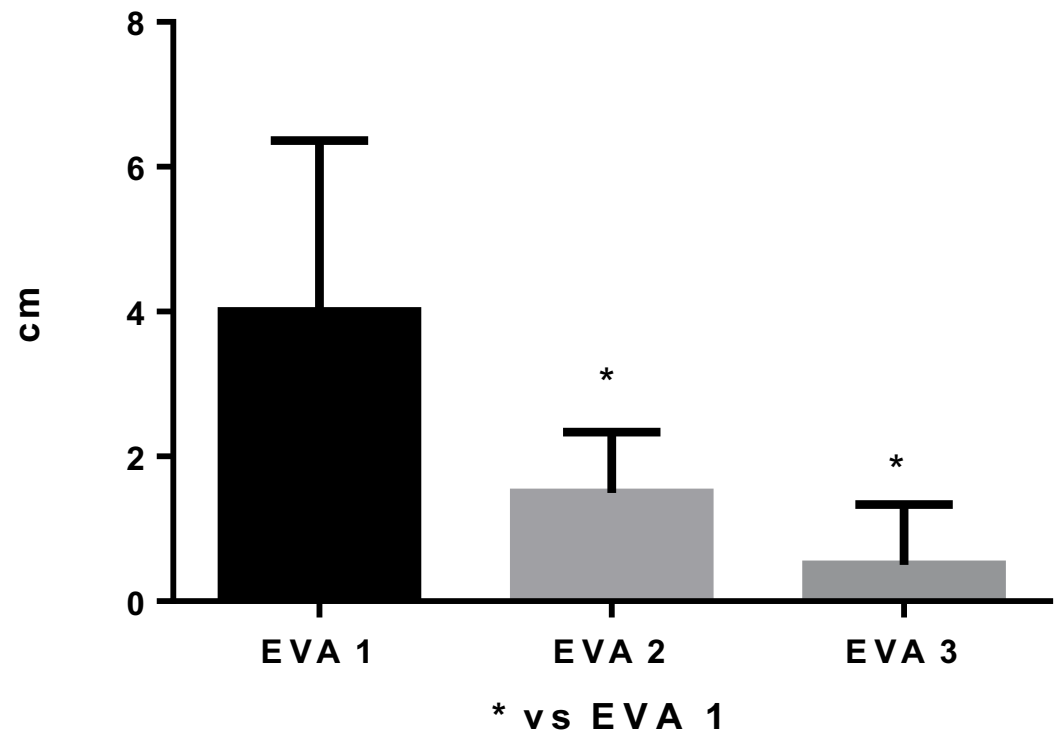

Fonte: Elaborado pelos Autores

(clique para voltar ao texto)

Figura 5 - Questionário Harris Hip Score Modificado, 2016

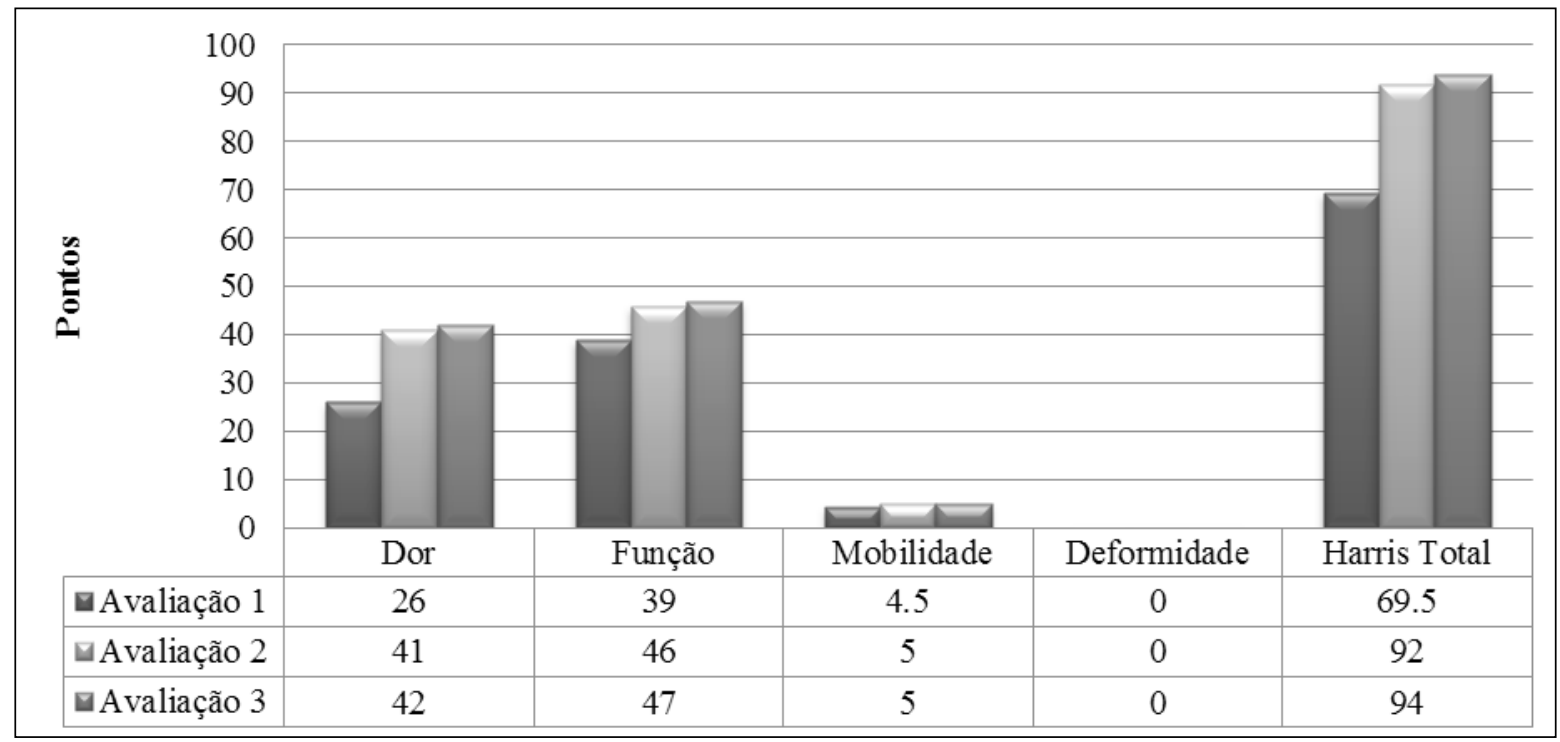

Fonte: Elaborado pelos autores

(clique para voltar ao texto)

\footnotetext{
${ }^{3}$ Valores apresentados com a média e desvio padrão na $1^{\mathrm{a}}$ semana (EVA 1), $4^{\mathrm{a}}$ semana (EVA 2) e na $8^{\mathrm{a}}$ semana de pós-operatório (EVA 3). *representa diferença estatística em relação à primeira avaliação (EVA 1).

${ }^{4}$ Valores apresentados com a média da dor, função, mobilidade, deformidade e Harris total na $1^{\text {a }}$ semana (avaliação 1), $4^{a}$ semana (avaliação 2) e na $8^{a}$ semana de pós-operatório (avaliação 3). *representa diferença estatística em relação à primeira avaliação.
} 